\title{
Correspondence
}

\section{The value of assessing suicidal ideation}

The paper by Gibbons et al concerning the distress experienced by psychiatrists who lose a patient through suicide is very welcome. We all know that such an event is difficult to bear, and this paper will encourage us to get support from others if things get really difficult, rather than soldiering on alone.

In describing how hard it is in clinical practice to detect suicide risk, let alone prevent it, the authors assert that recent research based on meta-analyses provides no evidence that suicide risk assessment in clinical practice can usefully guide clinical decision-making.

I believe that such a bald overall dismissal is regrettable because it discourages acquisition and critical evaluation of relevant clinical skills. Furthermore, I submit that it is not justified in the case of psychiatric in-patient care when addressing the problem of suicide prevention in the immediate or shortterm future.

Surely all would agree that in managing severe short-term risk we ignore evaluation of suicidal ideation at our peril. When less immediate short-term risk is considered, there is also much to affirm the important role of assessing suicidal ideation in guiding clinical decision-making.

We described two series of psychiatric in-patients (19821984, $N=27$; 1991-1993, $N=18$ ) who died by suicide either during hospital admission or within 2 months of discharge from hospital. ${ }^{2}$ In each of these, a high proportion of patients, 20/27 (74\%) and $15 / 18(83 \%)$, had discussed their suicidal ideas with members of staff in the ward during their in-patient stay. Suicidal ideation, as recorded contemporaneously in the case notes and not retrospectively, was a key clinical feature in delineating these patients, because they could not be distinguished from others in the ward across a range of behaviours. $12 / 27$ (44\%) and 9/18 (50\%) showed significant clinical improvement during their in-patient stay, even though stress in the community had remained unresolved. In the 10 years that had elapsed between our two series, the proportion of patients that killed themselves after discharge from hospital increased from $7 / 27$ (26\%) to $11 / 18$ (61\%).

These findings, based as they are on two small series of suicides, must be regarded as provisional. Yet they do suggest that knowledge of suicidal ideation can be useful in guiding clinical management decisions. Clinicians should be vigilant that clinical improvement in these particular patients may be temporary and misleading, possibly related to removal from stress in the community. They should be particularly careful to ensure that such stress has been resolved, or at least contained, by planned provision of adequate ongoing support in the community if discharge from hospital is envisaged. With the increased emphasis on community care, the proportion of these patients who kill themselves after discharge from hospital is likely to have increased further since our studies took place. Our findings suggest that clinicians should be mindful of the increased hazard which is likely to be associated with premature discharge of these patients from hospital.
Our in-patient psychiatric wards should be fertile ground for the necessary further research that is needed to clarify the many other possible aetiological factors in these suicidal deaths, and so help in the development of good clinical practice. The identification and evaluation of suicidal ideation must surely have a central role in this.

Gethin Morgan, Emeritus Professor of Mental Health, University of Bristol. Email: hilary.howard@blueyonder.co.uk

1 Gibbons R, Brand F, Carbonnier A, Croft A, Lascelles K, Wolfart G, Hawton K. Effects of patient suicide on psychiatrists; survey of experience and support required. BJPsych Bull 2019; 43: 241-63.

2 Morgan HG, Stanton R. Suicide among psychiatric patients in a changing clinical scene. Br J Psychiatry 1997; 171: 561-3.

doi:10.1192/bjb.2019.80

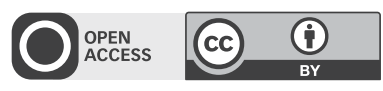

(c) The Author 2020. This is an Open Access article, distributed under the terms of the Creative Commons Attribution licence (http://creativecommons.org/ licenses/by/4.0/), which permits unrestricted re-use, distribution, and reproduction in any medium, provided the original work is properly cited

\section{Do foundation doctors meet the mental health competencies in the foundation programme curriculum?}

In recent years, the UK Foundation Programme has been adapted in order to meet the changing demands of patients, as well as those of foundation year doctors. A broadening of the Foundation Programme in 2014 stipulated that $100 \%$ of foundation year doctors should undertake a placement including community care from August 2017. ${ }^{1}$ Further to this, in 2015, Health Education England set a target that $45 \%$ of foundation year trainees should complete a psychiatry placement to gain exposure to mental healthcare. ${ }^{2}$ However, it is important to recognise that mental health problems do not present just in the psychiatry setting. General practice (GP) and accident and emergency (A\&E) are arguably 'gateways' to accessing mental healthcare in the National Health Service.

The Foundation Programme Curriculum 2016 outlines the expectations for what foundation doctors should learn. With the changes to community placements outlined above, I conducted a study to assess whether doing a foundation year placement in psychiatry, A\&E and/or GP affects trainees' ability to meet the Foundation Programme mental health competencies. I used a cross-sectional questionnaire to ask foundation year 2 (FY2) and CT1/ST1 trainees whether they felt they had met 17 mental health-related competencies from the curriculum. ${ }^{3}$ This was sent electronically via Foundation Schools.

A total of 360 trainees took the survey. Of all the trainees, only $29.7 \%(n=107)$ were aware that there are specific mental health competencies in the Foundation Programme curriculum 
that they should have met. Fourteen of the 17 competencies were self-assessed to have been met by trainees who completed a placement in psychiatry, compared with 15 out of 17 in $A \& E$ and 13 out of 17 in GP. By comparison, only nine competencies out of 17 were met by those who did not have a placement in any of the three specialties. Interestingly, in all groups, the competencies that should have been met by the end of FY1 were more successfully met than those for FY2.

These results were for trainees who may have taken one, two or all three specialties in their foundation year training. When considering each specialty uniquely, ten out of 17 competencies were met in psychiatry, whereas only six were met in $A \& E$ and nine in GP.

These results suggest that a combination of these three specialties may be more beneficial than one specialty alone, or none at all, in order for trainees to feel they have met the mental health competencies. There is also a case to be argued that trainees should be educated about the curriculum, as many were not aware they had the competencies to meet.

Further research should involve asking assessors to make the same competency assessments about the foundation year trainees, moving away from self-assessment to a work-based assessment.

\section{Obituary}

\section{Richard Green, MD, JD}

\section{Formerly Professor of Psychiatry, University College of Los Angeles, USA, and Visiting Professor of Psychological Medicine, Imperial College, London, UK}

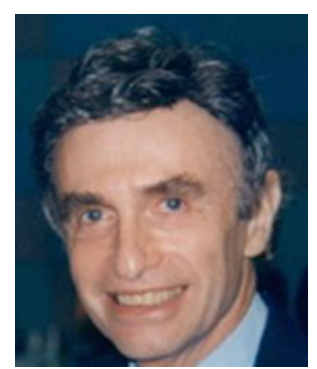

Throughout his life, Richard Green, who died on 6 April 2019, though heterosexual himself, acted as an effective advocate for gay men and women. In 1972, still in his 30s, he published a paper calling for the removal of homosexuality from the American Psychiatric Association's list of mental disorders. ${ }^{1}$ A year later, despite considerable opposition, this was achieved and there has been no subsequent attempt to reinstate it. Before this, in the 1960s, Green had worked with Harry Benjamin, who was seeing transgender patients. At that time surgical procedures to effect sex change could not be carried out in the USA. Green referred cases to Europe for sex reassignment.

From the 1960s he was active as an expert witness for gay men and women denied human rights on the grounds of their sexuality. In 1974, he appeared in a US television series advocating same-sex marriage. In the same year, he successfully
Jack Haywood, Academic Foundation Doctor, East and North Hertfordshire NHS Trust. email: jack.haywood@doctors.org.uk

1 Health Education England. Broadening the Foundation Programme: Recommendations and Implementation Guidance. HEE, 2014 (https:// www.hee.nhs.uk/sites/default/files/documents/Broadening\%20the\% 20Foundation\%20Programme\%20-\%20Recommendations\%20and\% 20implementation\%20guidance.pdf).

2 Perry J, Boyle A, Wessely S. The expansion of the Foundation Programme in psychiatry. BJPsych Bull 2016; 40(4): 223-5.

3 UK Foundation Programme. The Foundation Programme Curriculum 2016. UKFPO, 2015 (http://www.foundationprogramme.nhs.uk/sites/default/ files/2018-07/FP Curriculum_2016 V2\%20\%281\%29 0.pdf).

\section{doi:10.1192/bjb.2019.81}

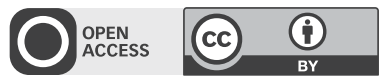

(c) The Author 2020. This is an Open Access article, distributed under the terms of the Creative Commons Attribution licence (http://creativecommons.org/ licenses/by/4.0/), which permits unrestricted re-use, distribution, and reproduction in any medium, provided the original work is properly cited.

pleaded that lesbian mothers should be allowed to have custody of their children in the event of a marriage break-up. Similarly, in 1979, he successfully supported a request from two gay Californian men that they should be allowed to co-adopt a child.

In addition to his successful legal advocacy, Green was responsible for groundbreaking research into homosexuality and transsexualism. He carried out highly significant, pioneering follow-up studies of effeminate boys and girls who were tomboys, establishing the extent to which they later developed a homosexual orientation. Among numerous publications, which include eight books and 200 papers and textbook chapters, Green co-edited the first multidisciplinary text (Transsexualism and Sex Reassignment ${ }^{2}$ ) on what was then commonly referred to as sex change. Green wrote about his career researching and advocating for people with homosexual orientation and transgender in his final book, Gay Rights, Trans Rights, self-published in $2018 .^{3}$

In 1971, he founded and edited the Archives of Sexual Behavior, which has remained the principal journal in the field. He continued as Editor until 2001. In 1974, he founded the International Academy of Sex Research (IASR) and was its first President. This has remained the principal academic society in the field and has an annual meeting that involves a programme of sex research-related presentations. Over the past 43 years, these meetings have been held in 18 different countries around the world.

Green was born in New York to secular Jewish parents, Leo (an accountant) and Rose Ingber (a teacher, later a civil servant). It is possible that his interest in homosexuality arose from the fact that, as he had no talent for sport, his father thought he was a sissy. After graduating with a BA from 